\title{
An electrophysiological dissociation of craving and stimulus-dependent attentional capture in smokers
}

\author{
Sarah E. Donohue ${ }^{1} \cdot$ Marty G. Woldorff $^{1,2} \cdot$ Jens-Max Hopf $^{1} \cdot$ Joseph A. Harris $^{1}$ • \\ Hans-Jochen Heinze $^{1}$ • Mircea A. Schoenfeld ${ }^{1,3}$
}

Published online: 24 August 2016

(C) Psychonomic Society, Inc. 2016

\begin{abstract}
It has been suggested that over the course of an addiction, addiction-related stimuli become highly salient in the environment, thereby capturing an addict's attention. To assess these effects neurally in smokers, and how they interact with craving, we recorded electroencephalography (EEG) in two sessions: one in which participants had just smoked (noncraving), and one in which they had abstained from smoking for $3 \mathrm{~h}$ (craving). In both sessions, participants performed a visual-search task in which two colored squares were presented to the left and right of fixation, with one color being the target to which they should shift attention and discriminate the locations of two missing corners. Task-irrelevant images, both smoking-related and non-smoking-related, were embedded in both squares, enabling the shift of spatial attention to the target to be examined as a function of the addiction-related image being present or absent in the target, the distractor, or both. Behaviorally, participants were slower to respond to targets containing a smoking-related image. Furthermore, when the target contained a smoking-related image, the neural responses indicated that attention had been shifted less
\end{abstract}

Electronic supplementary material The online version of this article (doi:10.3758/s13415-016-0457-9) contains supplementary material, which is available to authorized users.

Sarah E. Donohue

donohue.sarah.e@gmail.com

1 Department of Neurology and Leibniz Institute for Neurobiology, Otto-von-Guericke University Magdeburg, Leipziger Strasse 44, 39120 Magdeburg, Germany

2 Center for Cognitive Neuroscience, Department of Psychiatry and Behavioral Sciences, and Department of Psychology and Neuroscience, Duke University, Durham, NC, USA

3 Kliniken Schmieder Heidelberg Speyererhof, Heidelberg, Germany strongly to the target; when the distractor contained a smoking-related image, the shift of attention to the contralateral target was stronger. These effects occurred independently of craving and suggest that participants were actively avoiding the smoking-related images. Together, these results provide an electrophysiological dissociation between addiction-related visual-stimulus processing and the neural activity associated with craving.

Keywords Addiction $\cdot$ Attention $\cdot \mathrm{N} 2 \mathrm{pc} \cdot \mathrm{EEG} \cdot \mathrm{Craving} \cdot$ Smoking

Despite the well-known consequences that come with smoking, such as chronic disease and death, many people still regularly smoke cigarettes. The drive to smoke is largely based on craving for the next cigarette, and the state of craving can be so strong that it prevents people from quitting. Research into what drives these addictive behaviors has taken many different forms, including the examination of reward functioning (Koob \& LeMoal, 1997; Koob, Sanna, \& Bloom, 1998; Volkow \& Fowler, 2000), the study of inhibitory control (Perry \& Carroll, 2008; Volkow, Fowler, Wang, \& Swanson, 2004), and, more recently, the effect of attention on the processing of addiction-related stimuli (Field \& Cox, 2008). Much of this research has begun to provide what appears to be a complex picture of the behavioral and neural underpinnings of addiction; however, many facets of these processes still remain unknown, including how they may interact with craving.

One predominant theory as to what drives addiction is the "incentive sensitization" theory (Robinson \& Berridge, 1993). This theory posits that addiction-related substances are found to be rewarding upon use, and that such reward modifies the neural circuitry over time in a way that produces an attentional 
bias toward these stimuli, thereby enhancing craving for them (Robinson \& Berridge, 1993, 2008). Several behavioral studies have observed such addiction-related attentional capture, suggesting that there are indeed potential interactions between attentional bias and addiction. For example, when participants who smoke regularly are simultaneously presented with an image of a smoking-related stimulus and a neutral image in different locations on a screen, and are asked to respond to the location (or type) of a probe presented after both images disappear, participants are faster to respond to the probe if it occurs at the location where the smoking-related image had previously occurred (e.g., Ehrman et al., 2002; Mogg, Field, \& Bradley, 2005; Waters, Shiffman, Bradley, \& Mogg, 2003). This faster response in such visual-probe tasks has suggested that attention is more strongly present at the location of the addiction-related image, as would happen if participants had been cued to attend there (Posner, Snyder, \& Davidson, 1980). One key feature of these studies is that participants were never explicitly cued to attend to any image, and as such, any attentional capture observed was the result of the properties/ objects within the image itself that drew attention to that location (Field \& Cox, 2008). Findings such as these have been replicated across various substances of addiction, from cigarettes (e.g., Attwood, O'Sullivan, Leonards, Mackintosh, \& Munafò, 2008) to alcohol (e.g., Field, Mogg, Zetteler, \& Bradley, 2004), to opiates (e.g., Lubman, Peters, Mogg, Bradley, \& Deakin, 2000); however, such attentional capture is not consistently observed in all studies (see, e.g., Bradley, Mogg, Wright, \& Field, 2003, Exp. 1) and may depend on the level of substance dependence (Hogarth, Mogg, Bradley, Duka, \& Dickinson, 2003), the gender of the participants (Perlato, Santandrea, Libera, \& Chelazzi, 2014), and how such bias is measured across various types of addictions (Field \& Cox, 2008; Hendrikse et al., 2015; Leeman, Robinson, Waters, \& Sofuoglu, 2014).

If, indeed, such attentional capture by addiction-related stimuli is driven by the reward system, then one prediction would be that such attentional capture would be a rapid, automatic process. Colors and objects that have been associated with a monetary reward have been shown to capture attention in a rapid manner, enhancing (and sometimes speeding up) the rapid attentional shift toward those items, which occurs approximately $200 \mathrm{~ms}$ after the stimuli are presented in a search array (Donohue et al., 2016; Kiss, Driver, \& Eimer, 2009). Such reward-based attentional priority could drive the shift of attention to an addiction-related stimulus in a bottom-up manner (e.g., such that an addiction-related item will tend to "pop out" in a visual scene), thereby rendering the draw toward addiction-related stimuli automatic, and thus more daunting for addicts to overcome. The evidence is mixed, however, that attentional capture in addiction is in fact a rapid, automatic process. Some behavioral evidence suggests that attention is already drawn toward the addiction-related stimuli at very early (e.g., $50 \mathrm{~ms}$; Noël et al., 2006) time periods. Furthermore, at around $200 \mathrm{~ms}$, wherein reward-related attentional capture effects have been found (e.g., Donohue et al., 2016), two studies using smoking-related stimuli did find attentional capture by these images in smokers (Bradley, Field, Mogg, \& De Houwer, 2004; Chanon, Sours, \& Boettiger, 2010). Other studies, however, have not found such attentional capture at $200 \mathrm{~ms}$ (e.g., Bradley, Field, Healy, \& Mogg, 2008), or have found it only in male participants (Perlato et al., 2014), leaving the question open as to how automatic the capture of attention by addiction-related stimuli may be. The majority of studies examining attentional capture that have consistently found an attentional bias toward addiction-related stimuli have only tested longer stimulus-probe onset asynchronies (e.g., 500 or 2,000 ms; Attwood et al., 2008; Bradley, Garner, Hudson, \& Mogg, 2007; Bradley et al., 2003; Ehrman et al., 2002; Field, Mogg, \& Bradley, 2004; Mogg et al., 2005; Waters et al., 2003; see Field \& Cox, 2008, for a discussion), so it is unknown whether they would also have revealed a rapid capture of attention by these addiction-related stimuli.

Several studies have used electroencephalography (EEG) to examine the timing of the neural response to addictionrelated stimuli as compared to neutral images because this can offer a more precise marker of when attention may be captured than can behavior alone (e.g., Franken, Hulstijn, Stam, Hendriks, \& van den Brink, 2004; Littel \& Franken, 2007). The findings showed enhancements of slow-wave event-related potentials (ERPs), such as the P300 or the slow positive wave, to addiction-related stimuli, suggesting that attentional capture happens rather late in time (Franken et al., 2004; Littel \& Franken, 2007; see Littel, Euser, Munafò, \& Franken, 2012, for a review). However, as with the behavioral studies, findings of neural signatures of enhanced attentional capture are not always present, with one study failing to find a significant difference in attentional capture by smoking-related images for the P300 and the earlier P2a between smokers and nonsmokers (Bloom, Potts, Evans, \& Drobes, 2013). Indices of earlier electrophysiological signatures of attentional capture, such as the P1 and N1, have only been examined in one study using smokers and nonsmokers, and these components did not appear to differ across the groups (Littel \& Franken, 2011), although these components did differ across categories of images presented (e.g., smoking vs. positive images, for the P1). These early components (e.g., the P1) can be highly sensitive to the physical properties of the stimuli (e.g., luminance, spatial frequency; Ellemberg, Hammarrenger, Lepore, Roy, \& Guillemot, 2001; Hansen, Johnson, \& Ellemberg, 2012; Tobimatsu \& KuritaTashima, 1993), as well as to attention (Mangun, Buonocore, Girelli, \& Jha, 1998), and as such, it is unclear whether the lack of early modulation observed in the Littel and Franken (2011) study was due to physical differences between the stimuli overwhelming any differences that may have been 
related to attention, or whether attention simply would not be drawn to the smoking-related stimuli at such an early time period. Critically, of the aforementioned addiction-related ERP studies, only Littel and Franken (2007) explicitly controlled for the low-level physical characteristics of the stimuli used, and as such, any attentional capture reported in the other studies may have been due at least in part to these physical differences (e.g., luminance), as well as to the complexity of the stimuli themselves, rather than to the addiction-related properties of the stimuli per se.

In addition to the mixed evidence concerning whether and when addiction-related stimuli receive attentional priority, the manner in which the allocation of attention to such stimuli may interact with craving is even less clear (see Field, Munafò, \& Franken, 2009, for a review). When participants are presented with words or pictures related to their addiction, they often report an enhancement of their subjective amount of craving, as compared to when they are presented with neutral stimuli (e.g., Janes, Park, Farmer, \& Chakravarty, 2014; Lovett, Ham, \& Veilleux, 2015; Michalowski \& Erblich, 2014). This implies that exposure to addiction-related cues enhances craving. Franken (2003) proposed a model of addiction wherein attentional bias and craving can act in a reciprocal manner to enhance one another. However, some evidence suggests that this relationship between craving and attentional bias may not always be reciprocal. Specifically, when smokers performed both a modified smoking-related Stroop task and a visual-probe task examining behavioral responses to smoking-related versus neutral stimuli, a main effect of attentional bias toward the smoking-related stimuli was found; however, this effect did not interact with craving (Mogg \& Bradley, 2002). Nevertheless, a study using the visual-probe task in smokers who were either craving or not craving a cigarette revealed that, although the response time to the addiction-related probes did not interact with craving, craving participants did exhibit a longer dwell time on the smoking-related images, suggesting that visual focus can be influenced in some key ways by craving (Field, Mogg, \& Bradley, 2004).

Given the lack of a consensus as to how attentional bias and craving may interact in addiction, we sought to determine both the neural basis of the attentional capture by addiction-related images in smoking-addicted participants, and how such capture might interact with craving. To this end, we used EEG recordings of brain activity, a technique that allowed us to extract a neural marker of the shift of attention toward a smoking-related image on a millisecond timescale. In particular, we focused on an ERP component called the N2pc, which is a robust marker for the shift of attention to the spatial location of a stimulus, occurring approximately $200 \mathrm{~ms}$ after stimulus onset, contralateral to the location of the attentional shift (Eimer, 1996; Hopf et al., 2000; Luck \& Hillyard, 1994). The N2pc has been used to show reward-related attentional capture by colors (Kiss et al., 2009) and objects (Donohue et al., 2016), with these studies indicating that when participants shift attention in a search display toward reward-related items, this marker of attentional shift is both earlier in time and enhanced in amplitude. This specific component has previously not been used to determine the presence of attentional capture by addiction-related stimuli, but given that it can robustly indicate the shift of attention at an early time period, the N2pc would be particularly useful for detecting early signs of attentional capture in smokers.

To specifically assess the implicit capture of attention by addiction-related stimuli, we instructed participants to shift attention to a target of a specific color and to perform an orthogonal discrimination on the target. Embedded in the target and distractor stimuli were task-irrelevant images of either smoking-related or neutral items. Such a design allowed us to track the shift of attention to a color target and to determine how this process was modulated by the presence of a taskirrelevant smoking-related stimulus embedded in that target. Furthermore, participants completed the task twice, once when they had just smoked (non-craving), and once when they had not smoked for over $3 \mathrm{~h}$ (craving). If the shift of attention to addiction-related stimuli in smokers is an automatic process, then they should shift attention more rapidly to the target when the smoking-related images were embedded in it. Likewise, if the smoking-related images were embedded in the distractors, this should draw attention to them, which we should see in the form of an enhanced N2pc to the distractor. Furthermore, it is possible that this attentional bias might be enhanced under conditions of craving, since the drive to smoke under these circumstances would be higher, thereby tending to make the addiction-related stimuli more salient.

\section{Method}

\section{Subjects}

Data from 24 healthy participants were included in this study (14 male, 10 female, all right-handed, mean age $=25.2$ years, age range 22-31 years). The participants were prescreened for inclusion, and they all reported that they had smoked at least ten cigarettes per day for at least the past 6 months. Data were gathered from five additional participants, but they were excluded due to excessive physiological noise in the data (eye blinks and/or eye movements, $N=4$ ) or because the participant fell asleep $(N=1)$ in at least one of the two sessions. All experimental methods and procedures were approved by the Ethics Committee at the Otto-von-Guericke University of Magdeburg, and all participants gave written, informed consent prior to participation and were compensated for their time. 


\section{Craving manipulation}

Each participant took part in two experimental sessions. In one session, he or she had smoked approximately $45 \mathrm{~min}$ prior to the acquisition of neural data (the delay being due to the time it took to place the cap on the head and obtain a good connection between the scalp and the electrodes; see the detailed description below). In the other session, participants arrived $3 \mathrm{~h}$ prior to the experimental session and were closely monitored to ensure that they did not smoke during that time. They were therefore craving a cigarette by the time the neural data were recorded (approximately $3 \mathrm{~h} 45 \mathrm{~min}$ after their last cigarette). In both sessions, they performed the same visualsearch task, and the order of the sessions was randomized and counterbalanced across participants.

\section{Stimuli and task}

The experimental design was similar to that used by Donohue et al. (2016) with non-addicts. Each trial began with the presentation of two colored squares (one cyan and one yellow) located in the lower left and lower right quadrants (see Fig. 1). The central-most corner of each square was $1.72^{\circ}$ below and $2.64^{\circ}$ to the left or right of fixation. Each square subtended $3.3^{\circ} \times 3.3^{\circ}$ of visual angle. From each square two corners were missing, each of which subtended $0.77^{\circ} \times 0.77^{\circ}$ of visual angle; the corners could be either collinear or diagonal with respect to one another. The colors of the squares and the locations of the missing corners within the squares varied randomly from trial to trial, but the locations of the squares themselves remained constant throughout the experiment.

Prior to the start of the session, every participant completed a flicker-fusion task in which the luminances of the yellow and cyan colors used were equated. Each participant was then assigned a color to which he or she should attend (e.g., cyan) for both sessions. On each trial, the participants had to shift attention to the target color (for this example, to the cyan square located on either the left or the right side of the display) and determine whether the corners that were missing from that square were collinear or diagonal. The squares were displayed for $500 \mathrm{~ms}$, after which the fixation cross remained on the screen for $700 \mathrm{~ms}$. The participants responded with a button press using the index or middle finger of their right hand. For every trial, participants received performance feedback in the form of a 450-ms screen indicating whether or not their response was correct. Subsequently, the fixation cross remained on the screen for a period of time jittered between 200 and $400 \mathrm{~ms}$, after which the next trial began.

The key experimental manipulation was entirely task-irrelevant. Specifically, in order to determine the influence of the addiction-related stimuli on attentional capture, a grayscale image was presented within each colored square. These images were from either a nicotine-related product category (e.g., cigarettes, lighters) or an office-supply category (e.g., pens, a USB stick). Across categories, the images were equated for general physical similarity and mean luminance values, to avoid any low-level physical confounds that could attract attention to one category over another. In addition, any writing was removed from the objects to prevent the potential capture of attention due to written semantic content. Although keeping the stimuli physically similar may have slightly decreased the appeal of the smoking images, in this study we wished to test the attention-capturing properties of very basic addictionrelated stimuli. By minimizing the physical differences across the categories, we are able to determine that any effects we observed here were not due to categorical physical differences, but rather to the inherent meanings of the different objects to the smokers. Each category of objects had 40 different images, and each of these images was presented a total of 40 times (paired with either the target or the distractor color) over the course of the experiment. The pairings of the images with either the target or the distractor stimulus produced four different

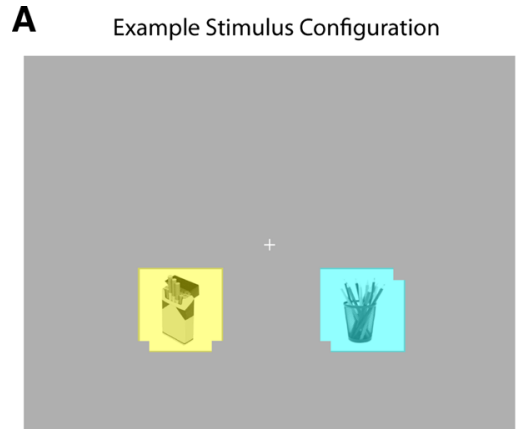

Fig. 1 (A) Example stimulus configuration. The participant had to shift attention to the attended color (e.g., cyan) and determine whether the missing corners were collinear or diagonal. Embedded within each square was an image that was either from a smoking-related image set or from an office-supply image set. Shown here is an example of a

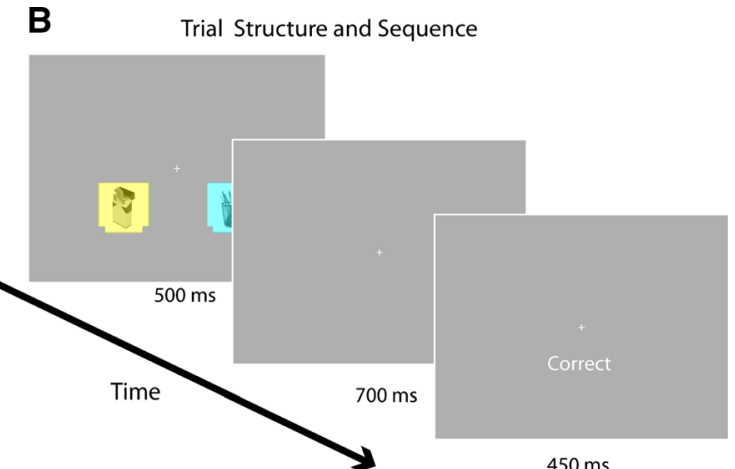

targOffice distSmoke trial. (B) Trial structure. Each trial began with the presentation of the search array, followed by a period of fixation. Feedback as to the accuracy of the response was subsequently presented on each trial. 
conditions: (1) smoking-related image in target position, officerelated image in distractor position (targSmoke_distOffice), (2) smoking-related images in both target and distractor (targSmoke_distSmoke), (3) office-related image in target, smoking-related image in distractor (targOffice_distSmoke), and (4) office-related images in both target and distractor (targOffice_distOffice).

Participants completed a practice block in each session prior to the start of the experiment, during which they were given feedback on their ability to maintain fixation and on their behavioral performance. Ten experimental blocks, each lasting just over $5 \mathrm{~min}$, were completed, for a total of 196 trials per experimental condition (e.g., TargetLeftSmoke_DistractorRightOffice). In addition, brief designated rest periods, during which participants were encouraged to blink, were inserted throughout the blocks. After the experimental session, resting-state data (not reported here) were acquired for each participant.

\section{Questionnaires}

Participants were administered the Fagerström Test for Nicotine Dependence (FTND) to determine their level of nicotine dependence (Heatherton, Kozlowski, Frecker, \& Fagerström, 1991). Just prior to collection of the neural data, participants were also given the Questionnaire on Smoking Urges (QSU) to determine their subjective level of craving through both positive symptoms ("desire and intention to smoke with an anticipation of pleasure from smoking") and negative symptoms ("the relief from nicotine withdrawal or the negative affect associated with an urgent and overwhelming desire to smoke"; Müller, Mucha, Ackermann, \& Pauli, 2001; Tiffany \& Drobes, 1991). Additional measures of reward-seeking and personality were obtained through the BIS/BAS questionnaire (Carver \& White, 1994). All versions of the questionnaires were administered in German, since that was the native language of the participants.

\section{EEG data acquisition}

Continuous EEG was recorded during both experimental sessions. The EEG data were acquired with a Neuroscan recording system (El Paso, Texas, USA). The EEG cap contained 32 channels (Easycap, Herrsching, Germany), including one channel placed below the right eye (VEOG) and one channel placed to the right of the right eye (HEOG) for detecting blinks and horizontal eye movements, respectively. The data were referenced online to the right mastoid, sampled at a rate of $508 \mathrm{~Hz}$, bandpass-filtered online from DC to $50 \mathrm{~Hz}$, and the impedance for each channel was maintained below $5 \mathrm{k} \Omega$.

\section{EEG data analysis}

The EEG data were divided in epochs offline, from -200 to $1,200 \mathrm{~ms}$ relative to stimulus onset, with only correct trials (i.e., those in which an accurate response occurred within a +200 - to 1,200 -ms post-stimulus time window) being retained for further processing. For each individual subject, peak-topeak artifact rejection thresholds were determined in a manner that was blind to the specific conditions. These thresholds, which ranged from 50 to $140 \mu \mathrm{V}$, resulted in a mean $17.2 \%$ of trials being rejected when the participants were not craving, and $19.2 \%$ when they were craving. These rejection percentages did not differ statistically from each other $[t(23)=1.16$, $p=.26]$. The data that remained after artifact rejection were then selectively averaged as a function of the various conditions and trial types. The ERP data were re-referenced to the average of the left and right mastoids. A grand average was then computed across participants separately for the craving and non-craving sessions. For plotting and statistical analysis, the data were baseline-corrected to the interval from -100 to $0 \mathrm{~ms}$ pre-stimulus.

P1 and N2pc extraction To determine the electrode sites for statistical testing for each of these effects in an unbiased manner, the ERP data were collapsed across all conditions and sessions. The visual-evoked response (the P1 component) was determined to be maximal (averaged across conditions and sessions) from 100 to $140 \mathrm{~ms}$ post-stimulus at sites PO3/PO4. The mean amplitudes for each condition and session were obtained for each participant at these sites for this time period, and these values were subjected to the statistical analyses described in the Results section below. This component was analyzed for the purpose of confirming that our physical controls for the categories of stimuli were sound. That is, if the categories of images used differed in a manner for which we were unable to control, the P1 component, which is sensitive to differences in such low-level properties as spatial frequency and luminance, would reflect as much.

The N2pc was extracted by subtracting those visual responses associated with right-visual-field targets from those associated with left-visual-field targets (separately for each condition and session). Averaged across conditions, this resulted in an N2pc effect that was maximal at sites PO7/PO8 during the post-stimulus time window of 175-275 ms.

\section{Results}

\section{Questionnaires}

The level of nicotine dependence, as measured with the FTND, varied across participants (score range: 1-8, mean $=4.67$ ). The mean BIS/BAS scores (and standard 
deviations) were as follows: BIS mean $=20.3(4.1)$, BAS Total $=40.9$ (5.3), BAS-Drive $=12.4$ (2.2), BAS-Fun Seeking $=12.2$ (2.2), BAS-Reward Responsiveness $=16.3$ (2.1). The craving manipulation was successful across participants since they reported significantly more craving, as measured by the QSU, for both the first factor, Positive Symptoms (see the Method section) [mean non-craving $=4.67$, mean craving $=5.84 ; t(23)=7.19, p<.001]$, and the second factor, Negative Symptoms [mean non-craving $=2.37$, mean craving $=3.25 ; t(23)=4.35, p<.001]$. The total QSU scores also significantly differed from each other as a function of craving $[$ mean non-craving $=3.64$, mean craving $=4.59 ; t(23)=8.35$, $p<.001]$. Interestingly, we found no correlation between the FTND and either of the QSU factors, suggesting that the level of overall nicotine addiction was not specifically related to the amount of craving that resulted from the craving manipulation in our experiment.

\section{Behavior}

The accuracy and response time (RT) data were submitted to two separate analyses of variance (ANOVAs), with the factors Craving (two levels: craving, non-craving), Smoking-Related Stimulus Present in the Target (two levels: present, not present), and Smoking-Related Stimulus Present in the Distractor (two levels: present, not present). Overall, participants were highly accurate in their judgments of the missing corners of the target squares (see Fig. 2A). Statistically, no significant main effects or interactions were observed [Craving, $F(1$, $23)=0.08, p=.78, \eta_{\mathrm{p}}{ }^{2}=.004$; Smoking-Related Stimulus in Target, $F(1,23)=0.94, p=.34, \eta_{\mathrm{p}}{ }^{2}=.04$; Smoking-Related Stimulus in Distractor, $F(1,23)=0.17, p=.72, \eta_{\mathrm{p}}{ }^{2}=.006$; Craving $\times$ Smoking-Related Stimulus in Target; $F(1,23)=$ $0.09, p=.77, \eta_{\mathrm{p}}{ }^{2}=.04 ;$ Craving $\times$ Smoking-Related Stimulus in Distractor, $F(1,23)=0.05, p=.82, \eta_{\mathrm{p}}{ }^{2}=.002$; Smoking-Related Stimulus in Target $\times$ Smoking-Related Stimulus in Distractor, $F(1,23)=0.15, p=.70, \eta_{\mathrm{p}}{ }^{2}=.007$; three-way interaction, $\left.F(1,23)=0.05, p=.83, \eta_{\mathrm{p}}{ }^{2}=.002\right]$. For the RT data, there was a significant main effect of the Smoking-Related Stimulus being Present in the Target, with the RTs to the target being slower when the smoking stimulus was present versus when it was absent $[F(1,23)=12.11$, $p=.002, \eta_{\mathrm{p}}{ }^{2}=.35$; see Fig. 2B]. No main effect of Craving emerged $\left[F(1,23)=0.04, p=.85, \eta_{\mathrm{p}}{ }^{2}=.002\right]$, there was no main effect of a Smoking-Related Stimulus being in the Distractor $\left[F(1,23)=0.50, p=.49, \eta_{\mathrm{p}}{ }^{2}=.02\right]$, and none of the interactions between the factors were significant [Craving $\times$ Smoking-Related Stimulus in Target, $F(1$, $23)=1.02, p=.32, \eta_{\mathrm{p}}{ }^{2}=.04 ;$ Craving $\times$ Smoking-Related Stimulus in Distractor, $F(1,23)=0.01, p=.92, \eta_{\mathrm{p}}{ }^{2}=.00$; Smoking-Related Stimulus in Target $\times$ Smoking-Related Stimulus in Distractor, $F(1,23)=0.86, p=.37, \eta_{\mathrm{p}}{ }^{2}=.04$; Craving $\times$ Smoking-Related Stimulus in Target $\times$ Smoking-
Related Stimulus in Distractor, $F(1,23)=0.03, p=.87$, $\left.\eta_{\mathrm{p}}{ }^{2}=.001\right]$. The manipulation of the embedded images, therefore, did not significantly impact behavioral accuracy, but having a smoking-related stimulus present in the target did increase the RT, regardless of whether or not the participants were craving a cigarette.

Early sensory-evoked component (P1) To determine whether any differences were present in the sensory-evoked visual responses to the onset of the stimuli, we examined the P1 component (see Fig. 3) in the ERP data. A repeated measures ANOVA was run on the mean amplitudes of this component from 100-140 ms, with the factors Craving (two levels: craving, non-craving), Electrode/Sensor Location (two levels: left hemisphere, right hemisphere), Target Location (two levels: left hemisphere, right hemisphere), Smoking-Related Stimulus Present in Target (two levels: present, not present), and Smoking-Related Stimulus Present in Distractor (two levels: present, not present). This analysis revealed a main effect of Craving, whereby the mean amplitude of the P1 was significantly larger when participants were craving than when they were not craving $\left[F(1,23)=10.54, p=.004, \eta_{\mathrm{p}}{ }^{2}=.31\right]$. No other significant effects or interactions were present (all $p s>.05$; see Table 1 for details of statistical output).

N2pc The N2pc was extracted by taking the response to the targets on the left side and subtracting from it the response to the targets on the right side, and then collapsing across the left and right sides for the traces and statistics, creating a difference between targets presented contralaterally versus ipsilaterally (see Supplementary Fig. 1 for the original, lateralized waveforms). This demonstrated a robust shift of attention contralateral to the target side (Fig. 4). To probe any effects of condition and/or craving on this shift of attention, the data that captured the N2pc (175-275 ms) were submitted to a repeated-measures ANOVA with the factors Craving (two levels: craving, non-craving), Smoking-Related Stimulus Present in Target (two levels: present, not present), and Smoking-Related Stimulus Present in Distractor (two levels: present, not present). This revealed a main effect of a Smoking-Related Stimulus Present in the Target $\left[F(1,23)=7.96, p=.01, \eta_{\mathrm{p}}{ }^{2}=.26\right]$, with the mean amplitude of the N2pc to the target being lower when the smoking stimulus was present than when it was absent, and a main effect of Smoking-Related Stimulus Present in the Distractor $[F(1$, $\left.23)=9.06, p=.006, \eta_{\mathrm{p}}{ }^{2}=.28\right]$, with the $\mathrm{N} 2 \mathrm{pc}$ to the target being higher when the smoking-related stimulus was in the distractor. We observed no main effect of Craving $[F(1,23)=$ $\left.2.79, p=.11, \eta_{\mathrm{p}}{ }^{2}=.11\right]$, and none of the interactions were significant [Craving $\times$ Smoking-Related Stimulus in Target, $F(1$, $23)=2.93, p=.10, \eta_{\mathrm{p}}{ }^{2}=.11$; Craving $\times$ Smoking-Related Stimulus in Distractor, $F(1,23)=0.77, p=.39, \eta_{\mathrm{p}}{ }^{2}=.03$; Smoking-Related Stimulus in Target $\times$ Smoking-Related Stimulus in Distractor, $F(1,23)=1.16, p=.29, \eta_{\mathrm{p}}{ }^{2}=.05$; 
Fig. 2 (A) Mean percentages correct across participants $(N=24)$. Overall, accuracy was high and did not differ significantly between the image conditions or as a function of craving. (B) Mean response times across participants $(N=24)$. The response times did not differ significantly as a function of craving; however, participants were slower overall to respond to the targets when a smoking-related image was present versus absent. In both panels, error bars represent the standard errors of the means.
A

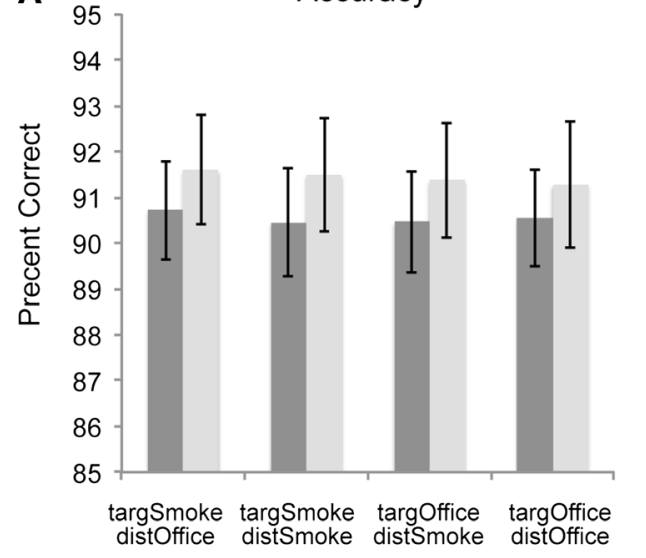

B

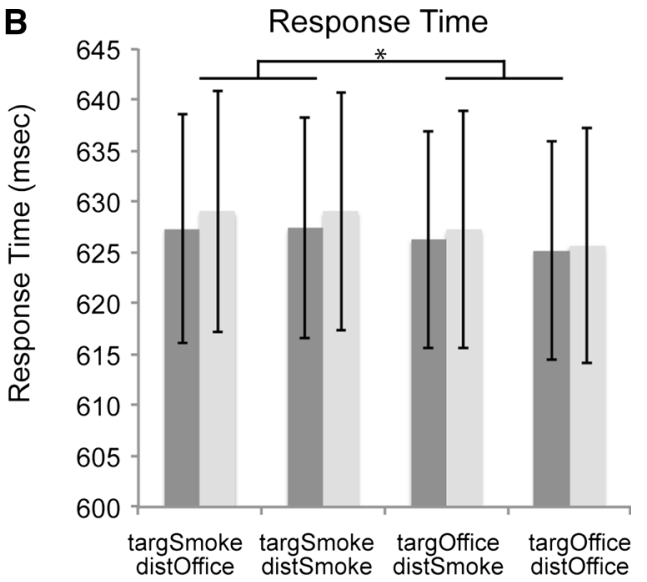

Craving $\times$ Smoking-Related Stimulus in Target $\times$ SmokingRelated Stimulus in Distractor, $F(1,23)=0.22, p=.64$, $\left.\eta_{\mathrm{p}}{ }^{2}=.01\right]$. As such, the shift of attention to the target was indeed modulated by the presence or absence of the addiction-related stimulus image, but these effects were not influenced by the participants' states of craving.

\section{Discussion}

In this study, we examined the neural reflections of addictionrelated attentional capture under conditions of craving and noncraving by using EEG recordings in 24 smokers. To this end, we used a task that allowed for the separation of the participants' task-related goal (a shift of attention to a colored target to make a discrimination about its missing corners) from the presentation of addiction-related stimuli, which were embedded in the target, in the distractor, in both the target and distractor, or were not present at all. Such a design allowed us to track how the capture of attention by addiction-related items modulated the attentional shift to, and subsequent processing of, the color target, relative to neutral non-smoking-related images. Behaviorally, participants were slower to respond to the corner detection task when a smoking-related image was present in the target than when it was absent from the target. In our neural measures, we observed that the shift of attention to a color target, as reflected by the N2pc, was enhanced when that target had a neutral image embedded in it relative to when it contained a smoking-related image, paralleling the behavioral effects. The shift of attention to the target was also modulated by the presence of a smoking-related image in the distractor, with an increased N2pc to the target when the addiction-related image was present in the distractor. Craving did not interact with the shift of attention to the target square or with the behavioral measures, but instead showed a more general modulatory enhancement of the early sensory-evoked P1 component, regardless of stimulus type. Thus, our results suggest that attention is not rapidly captured by, or at least drawn to, addiction-related stimuli in smokers. Indeed, nicotine-addicted participants appeared to direct their attention away from the smokingrelated stimuli. Moreover, these data provide evidence of a neural dissociation between the arousal effects of craving, reflected by modulation of the early sensory-evoked P1 component, and stimulus-related modulations of attentional shifting, reflected by effects on the N2pc and RTs.

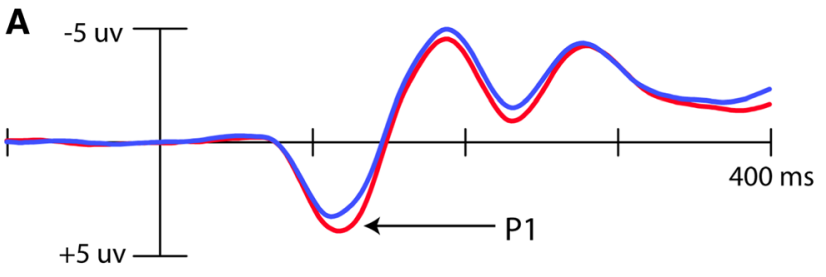

Craving Non-Craving
B

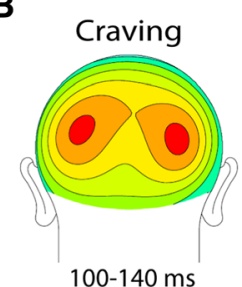

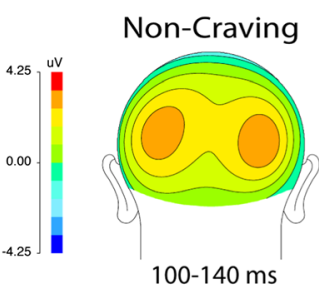

Fig. 3 P1 effect. (A) The P1 effect is shown for the event-related potential data averaged across sites $\mathrm{PO} 3$ and $\mathrm{PO} 4$. A larger P1 response was elicited when participants were craving than when they were not craving. (B) Topographic distributions of the P1 effect when participants were craving (left) versus non-craving (right). 
Table 1 Statistics for the P1 effect: $F$ values, $p$ values, and partial eta-squared values are reported from the output of the ANOVA run on the P1 results

\begin{tabular}{|c|c|c|c|}
\hline Main Effects and Interactions by Factors Used in ANOVA & $\begin{array}{l}F \\
\text { Value }\end{array}$ & $p$ & $\begin{array}{l}\text { Partial Eta- } \\
\text { Squared }\end{array}$ \\
\hline Craving & 10.54 & .004 & .31 \\
\hline Electrode & 0.22 & .64 & .01 \\
\hline Target Side & 3.98 & .06 & .15 \\
\hline Smoking in Target & 0.23 & .64 & .01 \\
\hline Smoking in Distractor & 0.15 & .71 & .006 \\
\hline Craving $\times$ Electrode & 0.20 & .66 & $<.01$ \\
\hline Craving $\times$ Target Side & 0.19 & 67 & $<.01$ \\
\hline Electrode $\times$ Target Side & 0.25 & 62 & .01 \\
\hline Craving $\times$ Electrode $\times$ Target Site & 0.25 & 62 & .01 \\
\hline Craving $\times$ Smoking in Target & 0.67 & .42 & .03 \\
\hline Electrode $\times$ Smoking in Target & 1.26 & .27 & .05 \\
\hline Craving $\times$ Electrode $\times$ Smoking in Target & 0.42 & .52 & .02 \\
\hline Target Side $\times$ Smoking in Target & 0.26 & 62 & .01 \\
\hline Craving $\times$ Target Side $\times$ Smoking in Target & 1.72 & .20 & .07 \\
\hline Electrode $\times$ Target Side $\times$ Smoking in Target & 0.45 & .51 & .02 \\
\hline Craving $\times$ Electrode $\times$ Target Side $\times$ Smoking in Target & 0.01 & .91 & $<.01$ \\
\hline Craving $\times$ Smoking in Distractor & 3.42 & .08 & .13 \\
\hline Electrode $\times$ Smoking in Distractor & 1.96 & .18 & .08 \\
\hline Craving $\times$ Electrode $\times$ Smoking in Distractor & 0.07 & .80 & $<.01$ \\
\hline Target Side $\times$ Smoking in Distractor & 0.95 & .34 & .04 \\
\hline Craving $\times$ Target Side $\times$ Smoking in Distractor & 0.13 & .72 & $<.01$ \\
\hline Electrode $\times$ Target Side $\times$ Smoking in Distractor & 0.49 & .49 & .02 \\
\hline Craving $\times$ Electrode $\times$ Target Side $\times$ Smoking in Distractor & 1.70 & .21 & .7 \\
\hline Smoking in Target $\times$ Smoking in Distractor & 0.25 & 62 & .01 \\
\hline Craving $\times$ Smoking in Target $\times$ Smoking in Distractor & 0.62 & .44 & .03 \\
\hline Electrode $\times$ Smoking in Target $\times$ Smoking in Distractor & 1.15 & .29 & .05 \\
\hline Craving $\times$ Electrode $\times$ Smoking in Target $\times$ Smoking in Distractor & 0.70 & .41 & .03 \\
\hline Target Side $\times$ Smoking in Target $\times$ Smoking in Distractor & 1.62 & .22 & .07 \\
\hline Craving $\times$ Target Side $\times$ Smoking in Target $\times$ Smoking in Distractor & 0.53 & .47 & .02 \\
\hline Electrode $\times$ Target Side $\times$ Smoking in Target $\times$ Smoking in Distractor & 1.54 & .23 & .06 \\
\hline $\begin{array}{l}\text { Craving } \times \text { Electrode } \times \text { Target Side } \times \text { Smoking in Target } \times \text { Smoking in } \\
\text { Distractor }\end{array}$ & 1.57 & .22 & .06 \\
\hline
\end{tabular}

Only the main effect of craving (in bold) reached significance.
The pattern of attentional-shift-related activity, as reflected by the $\mathrm{N} 2 \mathrm{pc}$, indicated that participants were shifting attention more strongly toward the office supplies, rather than toward the cigarettes. If one assumes that addiction-related stimuli carry a rewarding value, then this pattern of activity is in contrast to what has been seen in previous studies using rewarded items. Specifically, previous studies that have used rewarded colors (Kiss et al., 2009) and rewarded objects (Donohue et al., 2016) in visual-search tasks have shown a larger and earlier N2pc to these rewarded items. Not only did we not see such attentional bias occurring on this response, in the present study we observed the highest-amplitude modulations to the target when it contained a neutral office-supply image. Likewise, when a smoking image was present rather than absent in the distractor, the N2pc to the target was also greater, again suggesting an active avoidance of the smokingrelated image. The way in which attention is allocated in addiction contrasts with a reward-related attentional bias, in which items that are associated with a reward appear to automatically capture attention, even when they are not relevant for the current task (Anderson \& Yantis, 2011a, 2011b; Harris et al., 2016; Hickey, Chelazzi, \& Theeuwes, 2010; Qi, Zeng, Ding, \& Li, 2013). Here, perhaps, participants were invoking top-down control mechanisms to avoid potentially unwanted addiction-related images.

Key to the interpretation of the results is the understanding of what processes give rise to the N2pc. In a study conducted by Hickey and colleagues (2009), they found that the N2pc appears to comprise both the processing of a target (termed the $\mathrm{N}_{\mathrm{T}}$ ) and the processing of a distractor $\left(\mathrm{P}_{\mathrm{D}}\right)$. These processes 
A

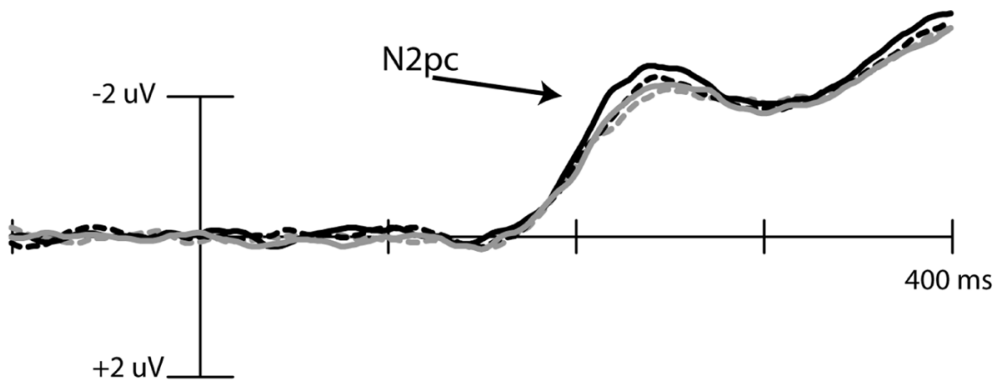

$$
\begin{array}{ll}
\ldots-\infty & \text { targSmoke_distOffice } \\
-\infty & \text { targSmoke_distSmoke } \\
- & \text { targOffice_distSmoke } \\
& \text { targOffice_distOffice }
\end{array}
$$

B

\section{Effects of Smoking-Related Stimulus in Target}

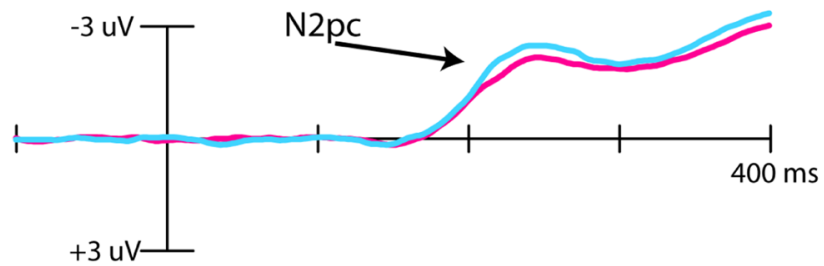

Effects of Smoking-Related Stimulus in Distractor

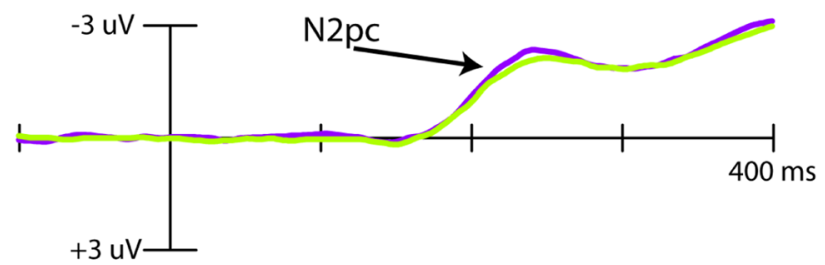

C

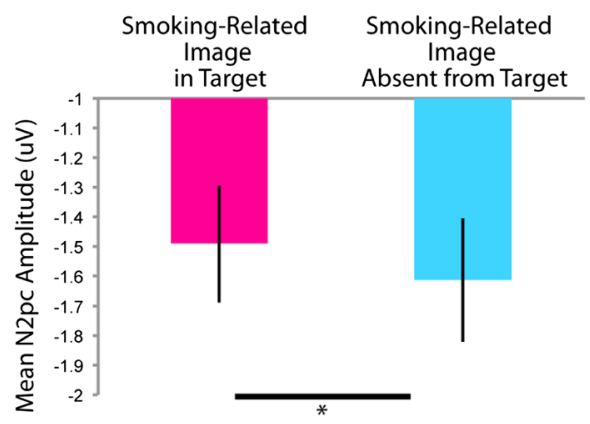

D
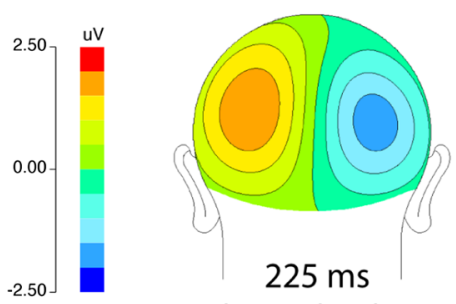

Smoking-Related Image in Target

Fig. 4 N2pc. (A) N2pc extracted for target left minus target right, shown for each condition. The largest N2pc to the target was elicited when the target stimulus contained an office supply and the distractor contained a smoking stimulus. (B) N2pcs extracted for target left minus target right, collapsed to reveal the difference between contralateral versus ipsilateral activity (left). Two main effects were present, one in which the N2pc to the target was larger when a non-smoking-related image versus a smoking-related image was present (left), and the other in which the

sum together, resulting in the activity observed in the N2pc, which reflects both the attentional selection of a target and the
-Smoking-Related Image in Distractor

_ Smoking-Related Image Absent from Distractor

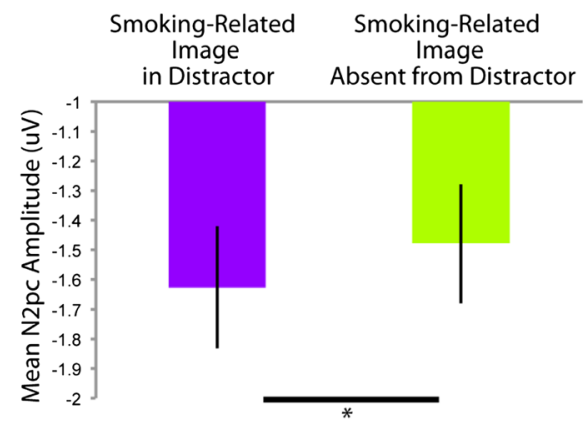

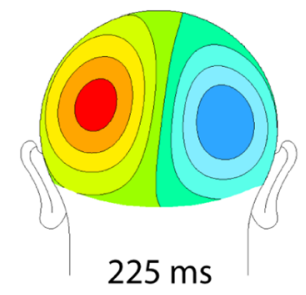

Smoking-Related Image in Distractor

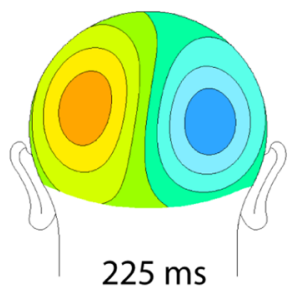

Smoking-Related Image absent from Distractor
$\mathrm{N} 2 \mathrm{pc}$ to the target was larger when a smoking-related image was present versus absent in the distractor (right). No differences in the $\mathrm{N} 2 \mathrm{pc}$ were observed as a function of craving. Sites shown are the rectified averages of PO7/PO8. (C) Mean amplitude plots of the main effects present in the N2pc from 175 to $275 \mathrm{~ms}$ as a function of a smoking image being present in the target (left) or in the distractor (right). Error bars represent the standard errors of the means. (D) Topographic distributions of the N2pc effects at 225 ms post-stimulus-onset.

suppression of a distractor. Although our present design was not optimized to pick up target selection and distractor 
suppression processes separately, we can, at least in a qualitative manner, assess the relative influences of these two processes. An examination of the traces in Fig. 4A shows that when the target is held constant (e.g., is a smoking-related image) and the distractor is either a smoking-related stimulus or an office supply, the amplitudes of the N2pc differ, suggesting an influence of the distractor processing on this component. Interestingly, the $\mathrm{N} 2 \mathrm{pc}$ to the target appeared to be larger when the distractor was a smoking-related image than when it was an office supply, likely indicating that the distractor-related suppression processing (i.e., the $\mathrm{P}_{\mathrm{D}}$ ) was larger under this circumstance. This bolsters the idea that the participants were trying to shift attention away from/ suppress smoking-related stimuli.

At first glance, it might seem that our attentional-bias effects are in contrast to those previously reported (e.g., Ehrman et al., 2002). It should be noted, however, that some key differences distinguish the paradigm presently used and previous work. First, the stimuli that we used were physically controlled and equated for luminance, to eliminate any bottomup physical differences from driving any image-related effects, whereas other studies either have not explicitly controlled for this (e.g., Versace et al., 2010), or have used stimuli that are far more complex (Littel \& Franken, 2007), and perhaps more "appealing" to smokers. Second, other studies observing such a bias toward attentional capture have either used tasks such as the addiction-related Stroop task, wherein the mechanisms of attentional bias may occur later in time (e.g., Munafò, Mogg, Roberts, Bradley, \& Murphy, 2003), or have observed such effects only when examining probe responses occurring over an extended time period, as late as 2,000 ms (e.g., Mogg et al., 2005). Because we did not examine such late time periods, we cannot say whether we would have also found evidence for late attentional capture by addictionrelated stimuli. Third, the time period during which some studies have observed an attentional bias (e.g., $500 \mathrm{~ms}$; Ehrman et al., 2002; Waters et al., 2003), actually falls within the timing of inhibition of return effects (see Klein, 2000, for a review). What this could imply is that, although participants in those studies did show an attentional bias toward the addiction-related stimuli, this could have been due to an initial direction of attention toward the non-addiction-related stimuli, followed by the direction of attention away from those images (i.e., toward the smoking ones) at $500 \mathrm{~ms}$. Fourth, the present study did not include a non-smoking group with which to compare the attentional capture results. Although our data do suggest an attentional modulation in the smokers by the addiction-related stimuli, the interpretation of the findings in the present study is limited by the lack of a direct comparison with non-smokers. Finally, our craving manipulation involved a smoking deprivation of only $3 \mathrm{~h}$, and thus it is unknown whether depriving the participants of smoking for a longer period would have resulted in different effects (e.g., 10+ h:
Field, Mogg, \& Bradley, 2004). Although there were significant differences between the QSU scores in the craving and non-craving sessions, which suggests that our craving manipulation was effective, it should be noted that in the noncraving session, by the time the EEG data were collected (and the questionnaire was filled out), some time had elapsed between that and when the participants had most recently smoked. This time was only $30-45 \mathrm{~min}$, but it is still possible that some participants were already starting to crave again, thereby making our non-craving condition not as "pure" as those in other studies in which participants had smoked immediately before the start of the experimental tasks (e.g., Mogg \& Bradley, 2002).

In the addiction literature, a bias similar to the one we have seen here has been reported in a behavioral study of alcoholic patients who were abstaining from drinking during in-patient therapy (Townshend \& Duka, 2007). These patients appeared to avoid alcohol-related stimuli, perhaps in an attempt to control their addiction. Moreover, other studies on alcohol use in both social drinkers and patients who have recently begun to abstain from alcohol suggest that although there may be some initial engagement by alcohol-related stimuli, the predominant response to these stimuli is avoidance (i.e., attentional disengagement) or the lack of an attentional bias (Gladwin, Ter Mors-Schulte, Ridderinkhof, \& Wiers, 2013; Noël et al., 2006; Vollstädt-Klein, Loeber, von der Goltz, Mann, \& Kiefer, 2009). Since our participants appeared to be avoiding the smoking-related images, they were presumably implementing top-down control mechanisms to do so. It is also possible that the capture of attention by these addiction-related stimuli in our participants had grown weaker over time. In a model of addiction proposed by Di Chiara (2000), the notion was put forth that as the addiction to a substance becomes stronger and more habitual, the attentional bias would grow weaker, a finding that has been observed in smokers (Mogg et al., 2005). Perhaps under the present circumstances, not only had the attentional bias grown weaker over time, but active control was also implemented throughout the duration of the experiment to avoid the addiction-related images, thereby resulting in the pattern of findings we observed here. Such an active control process could entail a predefined template for smoking-related images that, when one was detected, implemented a top-down mechanism to avoid such an image.

An interesting finding here is the role of craving (or lack thereof) in the processing of addiction-related stimuli. Strikingly, although we observed what could be interpreted as a withdrawal of attention from the smoking-related images (i.e., the shifting of attention toward the office-supply images on the other side), this effect was not modulated as a function of craving. These two processes therefore seem independent from one another, suggesting that craving does not drive attentional capture or attentional avoidance, at least at an early processing level. Since craving did modulate the P1, it is likely 
that in the craving condition participants may have had an overall greater level of attention present (Clark \& Hillyard, 1996; Luck, Heinze, Mangun \& Hillyard, 1990), perhaps due to heightened arousal, and that this transferred in a broad (i.e., nonspecific) manner to our task.

Another factor that could have influenced the results here is experimental demand. Specifically, all of the participants in this study were recruited because they were smokers, and as such, they were fully aware that this study was examining some facet of addiction (although the specific nature of the investigation was not explained until the post-experiment debriefing). It is possible, however, that because the participants knew this, they altered their behavior in such a way as to avoid the smoking-related images. Our effects occurred early in time $(\sim 200 \mathrm{~ms})$, and it is therefore not likely that participants were explicitly altering their behavior on a trial-by-trial basis by this time point (i.e., seeing a stimulus, processing it fully, then trying to avoid it due to wanting not to appear addicted), but such experimental demand characteristics may have played a more global role. Although we cannot rule this out as a possibility, the issue would be present for any study in which the participants were recruited on the basis of their addiction and shown addiction-related stimuli. As such, it is important to interpret these findings within the context of other studies that may also be susceptible to this factor.

In conclusion, our findings suggest that the capture of attention by an addiction-related image in smokers either does not automatically occur or is hindered by cognitive-control mechanisms, since we observed no evidence of such capture. If anything, participants were shifting their attention away from the smoking-related images, but doing so in a way that was not modulated by whether or not they were craving a cigarette. The effects of craving likely reflected overall arousal effects on the sensory processing of the visual stimuli, but the lack of any task-specific interaction suggested that such craving does not direct or shape the spatial attention toward an addiction-related stimulus. The results thus also suggest a dissociation between attentional-bias-related processes and craving effects in the human brain.

Author note We thank Steffi Bachmann for her extensive help with participant recruitment, data collection, and data entry. We also thank Stefan Knape for technical assistance. This work was supported by DFG Grant No. SFB 779 TPA14N.

\section{References}

Attwood, A. S., O'Sullivan, H., Leonards, U., Mackintosh, B., \& Munafò, M. R. (2008). Attentional bias training and cue reactivity in cigarette smokers. Addiction, 103, 1875-1882.

Bloom, E. L., Potts, G. F., Evans, D. E., \& Drobes, D. J. (2013). Cue reactivity in smokers: An event-related potential study. International
Journal of Psychophysiology, 90, 258-264. doi:10.1016/j. ijpsycho.2013.08.005

Bradley, B., Field, M., Healy, H., \& Mogg, K. (2008). Do the affective properties of smoking-related cues influence attentional and approach biases in cigarette smokers? Journal of Psychopharmacology, 22, 737-745.

Bradley, B., Field, M., Mogg, K., \& De Houwer, J. (2004). Attentional and evaluative biases for smoking cues in nicotine dependence: Component processes of biases in visual orienting. Behavioural Pharmacology, 15, 29-36.

Bradley, B. P., Garner, M., Hudson, L., \& Mogg, K. (2007). Influence of negative affect on selective attention to smoking-related cues and urge to smoke in cigarette smokers. Behavioural Pharmacology, 18, 255-263.

Bradley, B. P., Mogg, K., Wright, T., \& Field, M. (2003). Attentional bias in drug dependence: Vigilance for cigarette-related cues in smokers. Psychology of Addictive Behaviors, 17, 66-72.

Carver, C. S., \& White, T. L. (1994). Behavioral inhibition, behavioral activation, and affective responses to impending reward and punishment: The BIS/BAS Scales. Journal of Personality and Social Psychology, 67, 319-333. doi:10.1037/0022-3514.67.2.319

Chanon, V. W., Sours, C. R., \& Boettiger, C. A. (2010). Attentional bias toward cigarette cues in active smokers. Psychopharmacology, 212, 309-320.

Clark, V. P., \& Hillyard, S. A. (1996). Spatial selective attention affects early extrastriate but not striate components of the visual evoked potential. Journal of Cognitive Neuroscience, 8, 387-402. doi:10.1162/jocn.1996.8.5.387

Di Chiara, G. (2000). Role of dopamine in the behavioural actions of nicotine related to addiction. European Journal of Pharmacology, 393, 295-314.

Donohue, S. E., Hopf, J.-M., Bartsch, M. V., Schoenfeld, M. A., Heinze, H.-J., \& Woldorff, M. G. (2016). The rapid capture of attention by rewarded objects. Journal of Cognitive Neuroscience., 28, 529-541. doi:10.1162/jocn_a_00917

Ehrman, R. N., Robbins, S. J., Bromwell, M. A., Lankford, M. E., Monterosso, J. R., \& O’Brien, C. P. (2002). Comparing attentional bias to smoking cues in current smokers, former smokers, and nonsmokers using a dot-probe task. Drug and Alcohol Dependence, 67, $185-191$.

Eimer, M. (1996). The N2pc component as an indicator of attentional selectivity. Electroencephalography and Clinical Neurophysiology, 99, 225-234. doi:10.1016/0013-4694(96)95711-9

Ellemberg, D., Hammarrenger, B., Lepore, F., Roy, M. S., \& Guillemot, J. P. (2001). Contrast dependency of VEPs as a function of spatial frequency: The parvocellular and magnocellular contributions to human VEPs. Spatial Vision, 15, 99-111.

Field, M., \& Cox, W. (2008). Attentional bias in addictive behaviors: A review of its development, causes, and consequences. Drug and Alcohol Dependence, 97, 1-20. doi:10.1016/j. drugalcdep.2008.03.030

Field, M., Mogg, K., Zetteler, J., \& Bradley, B. P. (2004). Attentional biases for alcohol cues in heavy and light social drinkers: The roles of initial orienting and maintained attention. Psychopharmacology, 176, 88-93.

Field, M., Mogg, K., \& Bradley, B. P. (2004). Eye movements to smokingrelated cues: Effects of nicotine deprivation. Psychopharmacology, 173, 116-123.

Field, M., Munafò, M. R., \& Franken, I. H. A. (2009). A meta-analytic investigation of the relationship between attentional bias and subjective craving in substance abuse. Psychological Bulletin, 135, 589-607. doi:10.1037/a0015843

Franken, I. H. A. (2003). Drug craving and addiction: Integrating psychological and neuropsychopharmacological approaches. Progress in Neuro-Psychopharmacology and Biological Psychiatry, 27, 563579. 
Franken, I. H. A., Hulstijn, K. P., Stam, C. J., Hendriks, V. M., \& van den Brink, W. (2004). Two new neurophysiological indices of cocaine craving: Evoked brain potentials and cue modulated startle reflex. Journal of Psychopharmacology, 18, 544-552.

Gladwin, T. E., Ter Mors-Schulte, M. H. J., Ridderinkhof, K. R., \& Wiers, R. W. (2013). Medial parietal cortex activation related to attention control involving alcohol cues. Frontiers in Psychiatry, 4, 174. doi:10.3389/fpsyt.2013.00174

Hansen, B. C., Johnson, A. P., \& Ellemberg, D. (2012). Different spatial frequency bands selectively signal for natural image statistics in the early visual system. Journal of Neurophysiology, 108, 2160-2172.

Harris, J. A., Donohue, S. E., Schoenfeld, M. A., Hopf, J.-M., Heinze, H.J., \& Woldorff, M. G. (2016). Reward associated features capture attention in the absence of awareness: Evidence from objectsubstitution masking. NeuroImage, 137, 116-123. doi:10.1016/j. neuroimage.2016.05.010

Heatherton, T. F., Kozlowski, L. T., Frecker, R. C., \& Fagerström, K. O. (1991). The fagerström test for nicotine dependence: A revision of the fagerström tolerance questionnaire. British Journal of Addiction, 86, 1119-1127.

Hendrikse, J. J., Cachia, R. L., Kothe, E. J., McPhie, S., Skouteris, H., \& Hayden, M. J. (2015). Attentional biases for food cues in overweight and individuals with obesity: A systematic review of the literature. Obesity Reviews, 16, 424-432.

Hickey, C., Chelazzi, L., \& Theeuwes, J. (2010). Reward changes salience in human vision via the anterior cingulate. Journal of Neuroscience, 30, 11096-11103. doi:10.1523/JNEUROSCI.102610.2010

Hickey, C., Di Lollo, V., \& McDonald, J. J. (2009). Electrophysiological indices of target and distractor processing in visual search. Journal of Cognitive Neuroscience, 21, 760-775. doi:10.1162 /jocn.2009.21039

Hogarth, L. C., Mogg, K., Bradley, B. P., Duka, T., \& Dickinson, A. (2003). Attentional orienting towards smoking-related stimuli. Behavioural Pharmacology, 14, 153-160.

Hopf, J.-M., Luck, S. J., Girelli, M., Hagner, T., Mangun, G. R., Scheich, H., \& Heinze, H. J. (2000). Neural sources of focused attention in visual search. Cerebral Cortex, 10, 1233-1241.

Janes, A. C., Park, M. T. M., Farmer, S., \& Chakravarty, M. M. (2014). Striatal morphology is associated with tobacco cigarette craving. Neuropsychopharmacology, 40, 406-411.

Kiss, M., Driver, J., \& Eimer, M. (2009). Reward priority of visual target singletons modulates event-related potential signatures of attentional selection. Psychological Science, 20, 245-251. doi:10.1111/j.14679280.2009.02281.x

Klein, R. M. (2000). Inhibition of return. Trends in Cognitive Sciences, 4 , 138-147. doi:10.1016/S1364-6613(00)01452-2

Koob, G. F., \& LeMoal, M. (1997). Drug abuse: Hedonic homeostatic dysregulation. Science, 278, 52-58.

Koob, G. F., Sanna, P. P., \& Bloom, F. E. (1998). Neuroscience of addiction. Neuron, 21, 467-476.

Leeman, R. F., Robinson, C. D., Waters, A. J., \& Sofuoglu, M. (2014). A critical review of the literature on attentional bias in cocaine use disorder and suggestions for future research. Experimental and Clinical Psychopharmacology, 22, 469-483.

Littel, M., Euser, A. S., Munafò, M. R., \& Franken, I. H. A. (2012). Electrophysiological indices of biased cognitive processing of substance-related cues: A meta-analysis. Neuroscience \& Biobehavioral Reviews, 36, 1803-1816. doi:10.1016/j. neubiorev.2012.05.001

Littel, M., \& Franken, I. H. A. (2007). The effects of prolonged abstinence on the processing of smoking cues: An ERP study among smokers, ex-smokers and never-smokers. Journal of Psychopharmacology, 21, 873-882.
Littel, M., \& Franken, I. H. (2011). Implicit and explicit selective attention to smoking cues in smokers indexed by brain potentials. Journal of Psychopharmacology, 25, 503-513.

Lovett, D. E., Ham, L. S., \& Veilleux, J. C. (2015). Psychometric evaluation of a standardized set of alcohol cue photographs to assess craving. Addictive Behaviors, 48, 58-61.

Lubman, D. I., Peters, L. A., Mogg, K., Bradley, B. P., \& Deakin, J. F. (2000). Attentional bias for drug cues in opiate dependence. Psychological Medicine, 30, 169-175.

Luck, S. J., Heinze, H. J., Mangun, G. R., \& Hillyard, S. A. (1990). Visual event-related potentials index focused attention within bilateral stimulus arrays: II. Functional dissociation of P1 and N1 components. Electroencephalography and Clinical Neurophysiology, 75, 528542. doi:10.1016/0013-4694(90)90139-B

Luck, S. J., \& Hillyard, S. A. (1994). Electrophysiological correlates of feature analysis during visual search. Psychophysiology, 31, 291308.

Mangun, G., Buonocore, M., Girelli, M., \& Jha, A. (1998). ERP and fMRI measures of visual spatial selective attention. Human Brain Mapping, 6, 383-389.

Michalowski, A., \& Erblich, J. (2014). Reward dependence moderates smoking-cue- and stress-induced cigarette cravings. Addictive Behaviors, 39, 1879-1883.

Mogg, K., \& Bradley, B. P. (2002). Selective processing of smoking-related cues in smokers: Manipulation of deprivation level and comparison of three measures of processing bias. Journal of Psychopharmacology, 16, 385-392.

Mogg, K., Field, M., \& Bradley, B. P. (2005). Attentional and approach biases for smoking cues in smokers: An investigation of competing theoretical views of addiction. Psychopharmacology, 180, 333-341.

Müller, V., Mucha, R. F., Ackermann, K., \& Pauli, P. (2001). Die Erfassung des Cravings bei Rauchern mit einer deutschen Version des "Questionnaire on Smoking Urges" (QSU-G). Zeitschrift für Klinische Psychologie und Psychotherapie, 30, 164-171.

Munafò, M., Mogg, K., Roberts, S., Bradley, B. P., \& Murphy, M. (2003). Selective processing of smoking-related cues in current smokers, exsmokers and never-smokers on the modified Stroop task. Journal of Psychopharmacology, 17, 310-316.

Noël, X., Colmant, M., Van der Linden, M., Bechara, A., Bullens, Q., Hanak, C., \& Verbanck, P. (2006). Time course of attention for alcohol cues in abstinent alcoholic patients: The role of initial orienting. Alcoholism: Clinical and Experimental Research, 30, 1871-1877.

Perlato, A., Santandrea, E., Della Libera, C., \& Chelazzi, L. (2014). Biases of attention in chronic smokers: Men and women are not alike. Cognitive, Affective, \& Behavioral Neuroscience, 14, 742755. doi:10.3758/s13415-014-0287-6

Perry, J. L., \& Carroll, M. E. (2008). The role of impulsive behavior in drug abuse. Psychopharmacology, 200, 1-26.

Posner, M. I., Snyder, C. R., \& Davidson, B. J. (1980). Attention and the detection of signals. Journal of Experimental Psychology: General, 109, 160-174. doi:10.1037/0096-3445.109.2.160

Qi, S., Zeng, Q., Ding, C., \& Li, H. (2013). Neural correlates of rewarddriven attentional capture in visual search. Brain Research, 1532, $32-43$.

Robinson, T. E., \& Berridge, K. C. (1993). The neural basis of drug craving: An incentive-sensitization theory of addiction. Brain Research Reviews, 18, 247-291.

Robinson, T. E., \& Berridge, K. C. (2008). The incentive sensitization theory of addiction: Some current issues. Philosophical Transactions of the Royal Society B, 363, 3137-3146.

Tiffany, S. T., \& Drobes, D. J. (1991). The development and initial validation of a questionnaire on smoking urges. Addiction, 86, 14671476. doi:10.1111/j.1360-0443.1991.tb01732.x 
Tobimatsu, S., \& Kurita-Tashima, S. (1993). Effect of spatial frequency on transient and steady-state VEPs: Stimulation with checkerboard, square-wave grating and sinusoidal grating patterns. Journal of the Neurological Sciences., 118, 17-24.

Townshend, J. M., \& Duka, T. (2007). Avoidance of alcohol-related stimuli in alcohol-dependent inpatients. Alcoholism: Clinical and Experimental Research, 31, 1349-1357.

Versace, F., Robinson, J. D., Lam, C. Y., Minnix, J. A., Brown, V. L., Carter, B. L., Wetter, D. W., \& Cinciripini, P. M. (2010). Cigarette cues capture smokers' attention: Evidence from event-related potentials. Psychophysiology, 47, 435-441. doi:10.1111/j.14698986.2009.00946.x
Volkow, N. D., \& Fowler, J. S. (2000). Addiction, a disease of compulsion and drive: Involvement of the orbitofrontal cortex. Cerebral Cortex, 10, 318-325.

Volkow, N. D., Fowler, J. S., Wang, G.-J., \& Swanson, J. M. (2004). Dopamine in drug abuse and addiction: Results from imaging studies and treatment implications. Molecular Psychiatry, 9, 557-569.

Vollstädt-Klein, S., Loeber, S., von der Goltz, C., Mann, K., \& Kiefer, F. (2009). Avoidance of alcohol-related stimuli increases during the early stage of abstinence in alcohol-dependent patients. Alcohol and Alcoholism, 44, 458-463.

Waters, A. J., Shiffman, S., Bradley, B. P., \& Mogg, K. (2003). Attentional shifts to smoking cues in smokers. Addiction, 98, 1409-1417. 